\title{
Review of: "An Allometric Model Based Approach For Estimating Biomass In Seven Indian Bamboo Species In Western Himalayan Foothills, India"
}

Shuguang Wang

Potential competing interests: The author(s) declared that no potential competing interests exist.

This work was performed in order to develop more accurate model for the prediction of bamboo biomass, which could be useful for the bamboo farmers. However, some questions are still existed in the manuscript. In the Introduction part, the authors presented that the current questions is the presence of different ages, which influenced the biomass prediction. However, the authors said that the site specific predictive method is needed in the following sentences. The logicality is lack, and the importance and necessity need to be strengthened. 2. The disscussion parts also need to be strengthened. The authors considered that the ratio of dry to fresh weight was different in different organs and in different species, but why? Pls give the appropriate disscussion on it. Additionally, the authors also presented that different bamboo species had different prediction models. Pls discuss or present the possible reasons or explanation in the Disscussion parts. 